\title{
LncRNA MT1JP Suppresses Gastric Cancer Cell Proliferation and Migration Through MT1JP/MiR-214-3p/RUNX3 Axis
}

\author{
Ying Xu ${ }^{a}$ Guohua Zhang ${ }^{b}$ Chen Zou ${ }^{b}$ Heng Zhang ${ }^{b}$ Zhigang Gong ${ }^{b}$ \\ Weixin Wang ${ }^{\mathrm{b}}$ Gui Ma ${ }^{\mathrm{b}}$ Pengcheng Jiang ${ }^{\mathrm{b}}$ Wenbo Zhang ${ }^{\mathrm{b}}$ \\ aDepartment of Laboratory Center, Affiliated People's Hospital of Jiangsu University, Zhenjiang, \\ bDepartment of General Surgery, Affiliated People's Hospital of Jiangsu University, Zhenjiang, China
}

\section{Key Words}

Mt1jp • MiR-214-3p • RUNX3 • Proliferation • Migration • Gastric cancer

\begin{abstract}
Background/Aims: Emerging evidence points towards an important role of long noncoding RNAs (IncRNAs) in the tumorigenesis and progression of gastric cancer (GC). MT1JP has recently been reported to be differentially expressed and act as a tumor suppressor in different tumors, with its mechanisms not fully understood in GC. Methods: RT-qPCR was used to detect the expression of MT1JP, miR-214-3p and RUNX3 in tumor tissues and cell lines of GC. The CCK-8 assay, colony formation, Transwell assay and wound healing assay were used to evaluate the proliferation, invasion and migration of GC cells, respectively. Bioinformatics analysis and luciferase reporter assay were performed to disclose the interaction between MT1JP, miR-214-3p and RUNX3. Western blot and immunofluorescence were applied to assess the downstream signaling of RUNX3. Results: MT1JP was found downregulated in GC tissues and cells. Low expression of MT1JP was significantly correlated with advanced TNM stage and lymphatic metastasis. The expression of plasma MT1JP was also found decreased in GC patients compared to healthy controls, with an area under the ROC curve (AUC) of 0.649 for diagnosis of GC. Gain- and loss-of-function of MT1JP revealed that MT1JP functioned as a ceRNA for miR-214-3p to facilitate RUNX3 expression and then upregulated p21 and Bim levels suppressing GC cell proliferation, invasion and migration, and promoting apoptosis. Furthermore, MT1JP overexpression suppressed tumor growth and inhibited the expression of miR-214-3p and proliferation antigen Ki-67, but increased the expression of RUNX3, p21 and Bim in vivo. Conclusions: Our results suggest a potential ceRNA regulatory network involving MT1JP regulates RUNX3 expression by competitively binding endogenous miR214-3p in tumorigenesis and progression of GC. This mechanism may contribute to a better understanding of GC pathogenesis and provide potential therapeutic strategy for GC.
\end{abstract}

Y. Xu and G. Zhang contributed equally to this work. 


\section{Introduction}

Gastric cancer (GC) is the fourth most common malignancy and the second leading cause of global cancer-related death worldwide [1,2]. Despite advances in diagnostic markers and tools, the diagnosis of early stage GC is still relatively poor [3]. Thus, it is an urgent task for the identification of effective biomarkers for diagnosis and prognosis in GC.

Long noncoding RNAs (lncRNAs) are defined as transcripts that are longer than 200 nucleotides but do not appear to have protein-coding potential $[4,5]$. Cumulative evidence points towards an important role of IncRNAs in the regulation of tissue homeostasis and pathophysiological conditions [4, 6]. With the development of microarray and highthroughput screening as well as PCR, thousands of cancer-associated lncRNAs have been identifided recently [7, 8]. Functioned as oncogenes or tumor suppressors, IncRNAs participate in several vital steps of GC tumorigenesis and progression. Some lncRNAs including HOTAIR, HULC, LINC00152, MALAT1, MALAT2, H19, SNHG6, GHET1 and GACAT3 have been demonstrated to act as oncogenes, while other lncRNAs such as LEIGC, GAS5 and FER1L4 have been classified as tumor suppressors [9-12]. Mechanistically, lncRNAs may act as chromatin remodeler, transcriptional modulator, splicing regulator, posttranscriptional processor, enhancer, molecular decoys for miRNAs, or as a guide for protein-protein, proteinDNA and protein-RNA interaction $[4,5,13,14]$. Given the properties of disease-, tissue- and developmental-specificity, lncRNA may serve as a candidate biomarker for GC diagnosis [9, $13,15]$. Indeed, recent evidences have confirmed that several IncRNAs may not only provide significance for GC diagnosis, stage assessment and prognosis prediction, but also serve as promising targets for GC treatment $[9,13,15]$.

LncRNA MT1JP resides on chromosome 16 in a cluster consisting of several homologous protein-coding genes of the metallothionein family. Using microarray analysis, MT1JP has previously been shown downregulated in several tumors [16]. Knockdown of MT1JP results in a range of changes which usually arise during tumor formation, including cell cycle arrest, apoptosis inhibition, proliferation acceleration, and increased invasion and migration, as opposed to the effects of MT1JP overexpression [16]. These results suggests that MT1JP is necessary for maintaining the normal life activities of cells, and that it may have a critical function as a tumor suppressor. Nonetheless, little is known regarding the biological role of MT1JP in the process of GC. Based on the emerging theory of competing endogenous RNAs (ceRNAs), we hypothesized that MT1JP might inhibit GC progression through inhibition of miR-214-3p expression. To this end, we first detected the expression of MT1JP and miR214-3p in tumor tissues of GC as well as in GC cells. Furthermore, the potential downstream signaling pathway of MT1JP in the development of GC was analyzed both in vitro and in vivo. Therefore, this study may contribute to a better understanding of GC pathogenesis and provide potential therapeutic strategy for GC.

\section{Materials and Methods}

\section{Patients and specimens}

Fresh GC tissues and paired noncancerous tissues from patients with GC were collected at the Affiliated People's Hospital of Jiangsu University from 2014 to 2016. Informed consent for the use of samples was obtained from all patients. All samples were diagnosed by 2-3 pathologists blinded to the patients. Inclusion criteria were patients with primary GC stages I-IV, having undergone surgery as the initial treatment modality and having complete clinicopathological data. None of the patients had received preoperative therapies. Plasma samples were collected from each subject as well as 34 healthy donors included in the same period, and stored at $-80^{\circ} \mathrm{C}$ before use. This study was approved by the Institutional Ethical Committee of Jiangsu University. Tumor stage was evaluated according to the TNM classification system of the International Union Against Cancer ( $7^{\text {th }}$ edition, 2010).

\section{KARGER}


Cell culture and transfection

The human GC cell lines SGC-7901, MGC-803, HGC-27 and AGS were purchased from the Cell Bank of the Chinese Academy of Sciences (Shanghai, China). The human normal gastric epithelial cell line GES-1 was obtained from Procell Life Science \& Technology (Wuhan, China). Human embryonic kidney (HEK)-293T was from American Type Culture Collection (Manassas, VA, USA). MGC-803 and HEK-293T was cultured with high glucose-DMEM (Thermo Fisher Scientific, MA) containing 10\% fetal bovine serum (FBS; Gibco) and a $100 \mathrm{U} / \mathrm{ml}$ penicillin/streptomycin mixture at $37{ }^{\circ} \mathrm{C}$ in $5 \% \mathrm{CO}_{2}$. GES-1, HGC-27 and SGC-7901 were cultured with RPMI 1640 medium (Thermo). AGS was cultured with DMEM/F-12 medium (Thermo).

\section{Animals}

Nude mouse (4weeks, male) were purchased from Shanghai Laboratory Animal Center (SLAC, Shanghai, China). All animals were maintained under specific pathogen free condition at constant room temperature $\left(21 \pm 2^{\circ} \mathrm{C}\right)$ and allowed to water and food ad libitum in $12 \mathrm{~h}$ dark/light cycle. The experimental protocol was approved by the Institutional Animal Care and Use Committee of Jiangsu University in China. All animal experiments were conducted in conformance with the Guide for the Care and Use of Laboratory Animals published by the US National Institutes of Health (NIH Publication, 8th edition, 2011).

\section{RNA isolation and Reverse Transcription Quantitative Polymerase Chain Reaction (RT-qPCR)}

Total RNA was extracted from tissues, serums and different GC cells using TRIzol Reagent (Sangon Biotech, Shanghai). After concentration, purity and integrity detections, $1 \mu \mathrm{g}$ of DNase I-treated RNA was applied in the reverse transcription reaction with a Hieff First Strand cDNA Synthesis Super Mix for RT-qPCR kit (Yeasen Biotech, Shanghai). Quantitative PCR analysis was carried out using the Hieff qPCR SYBR Green Master Mix kit (Yeasen Biotech, Shanghai) on an ABI Prism 7300 Sequence Detection System (Applied Biosystems, CA). The primer sequences are listed as follows: MT1JP forward: 5'-GCAAAGGGACGTCGGAGA-3', reverse: 5'-TCCAGGTTGTGCAGGTTGTT-3', RUNX3 forward: 5'-CCACTCTGCTCTGTCAGCTT-3', reverse: ATTTGGATTCCCGGTCCCAC-3', p21 forward: 5'-CCCGTGAGCGATGGAACTTC-3', reverse: 5'-CCCGTGGGAAGGTAGAGCTT-3', Bim forward: 5'-GCAGTTTGTTGGAGCTCTGC-3', reverse: 5'-AGGGTGGCTGCAAGAATCAA-3', miR-214-3p forward: 5'-ACAGCAGGCACAGACAGGCA-3', miR-24-3p forward: 5'-CTGGCTCAGTTCAGCAGGAACAG-3', miR-30c-2-3p forward: 5'-GCTGGGAGAAGGCTGTTTACTCT-3', miR-185-5p forward: 5'-GTGGAGAGAAAGGCAGTTCCTGA-3', miR-298 forward: 5'-AGCAGAAGCAGGGAGGTTCTCC-3', miR-4863p forward: 5'-GGGGCAGCTCAGTACAGGAT-3', universal reverse primer: 5'-AGTGCAGGGTCCGAGGTATT-3', $\beta$-actin forward: 5'- CATTCCAAATATGAGATGCGTTGT-3', reverse: 5'-TGTGGACTTGGGAGAGGACT-3', U6 forward: 5'-CTCGCTTCGGCAGCACA-3', reverse: 5'-AACGCTTCACGAATTTGCGT-3'. $\beta$-actin or U6 was used as an endogenous control depending on the gene/sample detected. Relative expression levels were calculated by using the $2^{-\Delta \Delta C t}$ method.

\section{Constructs, synthesized oligos and transfection}

The MT1JP overexpression plasmid was purchased from GenePharm (Shanghai, China). Briefly, MT1JP sequence was amplified and inserted into the pcDNA3.1 (+) vector at the BamH1 sites. The primers used were as follows: forward: 5'-TACCGAGCTCGGATCCTTGCGGTCTCTCCATTTATCG-3', reverse: 5'-CGTTACTAGTGGATCCGCAGCAAATGGCTCAGTATTG-3'. The MT1JP siRNAs, RUNX3 siRNAs, miR-214$3 p$ mimics and anti-miR-214-3p, as well as scrambled negative control oligos, were also synthesized by GenePharm. For transfection, the cells were grown in 12-well plate until confluence at 60-80\%, and were transfected with the indicated molecules with Lipofectamine 2000 (Thermo) according to the manufacturer's instructions. Forty-eight hours after transfection, the cells were ready for the following experiments.

\section{Proliferation and apoptosis}

The cell proliferation assay was performed using a Cell Counting Kit-8 kit (Vazyme Biotech, Nanjing) according to the manufacturer's instructions. The absorbance at $450 \mathrm{~nm}$ was recorded and analyzed. For cell apoptosis assay, the indicated cells were stained with Annexin V-FITC/PI cell apoptosis detection kit (BD Pharmingen, San Diego, CA, USA) and were analyzed with a flow cytometer (BD FACSCalibur, San Jose, CA, USA)

\section{KARGER}




\section{Cellular Physiology Cell Physiol Biochem 2018;46:2445-2459

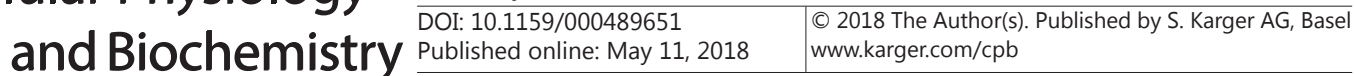

Xu et al.: MT1JP Regulates GC through miR-214-3p/RUNX3

Invasion assay

Invasion assay were performed in Transwell chambers with Matrigel-coated membranes. Briefly, GC cells were seeded with serum-free medium into the upper chambers at $5 \times 10^{4}$ cells/well, and the bottom chambers contained medium with 10\% FBS. After a 24-h incubation, nonmigrating cells on the upper surface of the membrane were removed with a cotton swab, and cells penetrating to the lower surface of the membrane were stained with crystal violet. Cell numbers were counted in five randomly chosen microscopic fields per membrane.

\section{Wound healing assays}

The cells were seeded in six-well plates until confluence. Streaks across the plate were created in the monolayer with a pipette tip, followed by 3 washes in serum-free medium. The cells were then cultured for $24 \mathrm{~h}$ with medium containing 1\% FBS. To ensure documentation of the same region, the wells were marked across the wounded area. Progression of migration was observed and photographed at 0 and $24 \mathrm{~h}$ after wounding. The distance between the two edges of the scratch was measured and calculated.

\section{Luciferase constructs and activity detection}

The luciferase constructs were purchased from GenePharm (Shanghai, China). Briefly, the 3'UTR of RUNX3 or MT1JP transcript were cloned into the downstream of luciferase gene to generate pGL3-RUNX3WT or pGL3-MT1JP-WT vector. Constructs with site-directed mutations of the putative binding sequences were prepared by overlapping PCR. HEK-293T cells were then transfected with the indicated luciferase vectors as described in the corresponding figure legends. The luciferase activities were measured $48 \mathrm{~h}$ after transfection using the dual-luciferase assay kit (Promega, Madison, WI, USA) according to the manufacturer's protocol.

\section{Subcellular fractionation}

Cytoplasmic and nuclear fractions were isolated with NE-PER ${ }^{\mathrm{TM}}$ Nuclear and Cytoplasmic Extraction Reagents (Thermo) according to the manufacturer's instructions. RNA or protein was extracted from each fraction, and was determined by RT-qPCR or Western blot analysis, respectively.

\section{Western blot}

Equal amounts of protein samples were separated by 8-15\% SDS-PAGE and transferred to nitrocellulose membranes. Bands were probed immunologically using anti-RUNX3 (Pathway Biological Technology, Nanjing), p21 (Sangon Biotech, Shanghai), Bim (Sangon Biotech), GAPDH (Cell signaling, MA, USA), $\beta$-actin (Cell signaling) and Histone H3 (Cell signaling). Signals were detected using an ECL system according to the manufacturer's instructions. Intensities of the immunoreactive bands were determined using Image LabTM software (version 4.1, Bio-Rad, CA).

\section{Immunofluorescence}

For immunofluorescence, frozen tissue sections or cell slides were permeabilized with $0.3 \%$ Triton X-100, blocked with 3\% normal goat serum for $30 \mathrm{~min}$, and incubated with anti-RUNX3 (Pathway Biological Technology), Ki-67 (Santa Cruz, CA), p21 (Sangon Biotech) or Bim (Sangon Biotech) overnight. The sections or slides were then incubated with anti-rabbit Alexa Fluor 488 (Jackson Immunoresearch, West Grove, PA, USA) or anti-mouse Alexa Fluor 594 (Jackson Immunoresearch), accordingly. DAPI was used for nuclear counterstaining. The samples were observed under a fluorescence microscope (Leica, Mannheim, Germany). Positive cells were counted blindly in 10 high power field $(\mathrm{HPF}) /$ section $(\times 200)$.

\section{Tumor xenograft experiment}

HGC-27 cells stably expressing empty vector or pcDNA-MT1JP were selected by G418 (Invitrogen, Carlsbad, CA). The indicated cells $\left(100 \mu \mathrm{l}, 2 \times 10^{6}\right)$ were inoculated subcutaneously into the dorsal right flank of each nude mouse ( 6 mice per group). Tumor volumes were calculated as $0.5 \times$ length $\times$ width $^{2}$ every 3 days. After 4 weeks, mice were sacrificed, and tumors were excised, weighed and immediately frozen at $-80^{\circ} \mathrm{C}$ for future use. 
Statistical analysis

All statistical analysis were conducted with SPSS ${ }^{\circledR}$ version 22.0 (SPSS, Chicago, USA). Differences between two dependent groups were evaluated with the Student's t-test. Comparisons among multiple groups were performed with one-way ANOVA followed by Bonferroni post-hoc tests. The associations between MT1JP and the clinicopathological features were analyzed by the Pearson $\chi^{2}$ test. The receiver operating characteristic (ROC) curve was established to evaluate the diagnostic value of MT1JP for GC. All $P$-values were two-tailed, and $P<0.05$ was accepted as being statistically significant.

\section{Results}

\section{Correlation of MT1JP expression with} clinicopathological features

To explore the expression profile of MT1JP in GC, we first detected MT1JP expression levels in 99 pairs of GC tissues and neighboring noncancerous tissues by RT-qPCR. As shown in Fig. 1A, the transcript levels of MT1JP were significantly lower in GC tissues compared with neighboring noncancerous tissues from the same patient. Additionally, the expression of MT1JP was downregulated in $68.7 \%$ (68/99) of GC tissues compared to that in the adjacent normal tissues (Fig. 1B). We further found that 41 cases displayed at least one-half decrease in GC tissues compared to the paired non-tumor tissues. We then stratified the patients into low expression group $(\mathrm{n}=41$, fold change $\leq 0.5)$ and high expression group $(n=58$, fold change $>0.5)$. As shown in Table 1 and Fig. 1C, downregulation of MT1JP in GC tissues was correlated with lymphatic metastasis and advanced TNM stage. In addition,
Table 1. Correlation between MT1JP expression and patients' clinicopathological characteristics

\begin{tabular}{|c|c|c|c|c|}
\hline \multirow{2}{*}{ Feature } & \multirow{2}{*}{ Number } & \multicolumn{2}{|c|}{ MT1JP expression } & \multirow{2}{*}{ P value } \\
\hline & & & & \\
\hline \multicolumn{5}{|l|}{ Gender } \\
\hline Male & 78 & 46 & 32 & \multirow[t]{2}{*}{0.880} \\
\hline Female & 21 & 12 & 9 & \\
\hline \multicolumn{5}{|l|}{ Age } \\
\hline$<60$ & 19 & 12 & 7 & \multirow[t]{2}{*}{0.653} \\
\hline$\geq 60$ & 80 & 46 & 34 & \\
\hline \multicolumn{5}{|l|}{ Drink } \\
\hline No & 76 & 45 & 31 & \multirow[t]{2}{*}{0.819} \\
\hline Yes & 23 & 13 & 10 & \\
\hline \multicolumn{5}{|l|}{ Smoke } \\
\hline No & 70 & 40 & 30 & \multirow[t]{2}{*}{0.651} \\
\hline Yes & 29 & 18 & 11 & \\
\hline \multicolumn{5}{|l|}{ Tumor size } \\
\hline$<5 \mathrm{~cm}$ & 51 & 34 & 17 & \multirow[t]{2}{*}{0.092} \\
\hline$\geq 5 \mathrm{~cm}$ & 48 & 24 & 24 & \\
\hline \multicolumn{5}{|c|}{ Differentiation } \\
\hline Moderate & 38 & 19 & 19 & \multirow[t]{2}{*}{0.171} \\
\hline Poor & 61 & 39 & 22 & \\
\hline \multicolumn{5}{|l|}{ Location } \\
\hline Proximal & 42 & 25 & 17 & \multirow[t]{3}{*}{0.717} \\
\hline Middle & 32 & 20 & 12 & \\
\hline Distal & 25 & 13 & 12 & \\
\hline \multicolumn{5}{|c|}{ Lymphatic metastasis } \\
\hline Negative & 22 & 18 & 4 & \multirow[t]{2}{*}{0.014} \\
\hline Positive & 77 & 40 & 37 & \\
\hline \multicolumn{5}{|c|}{ Venous invasion } \\
\hline Negative & 62 & 37 & 25 & \multirow[t]{2}{*}{0.775} \\
\hline Positive & 37 & 21 & 16 & \\
\hline \multicolumn{5}{|c|}{ Invasion depth } \\
\hline T1-2 & 7 & 5 & 2 & \multirow[t]{2}{*}{0.696} \\
\hline T3-4 & 92 & 53 & 39 & \\
\hline \multicolumn{5}{|l|}{ M } \\
\hline M0 & 97 & 56 & 41 & \multirow[t]{2}{*}{0.510} \\
\hline M1 & 2 & 2 & 0 & \\
\hline \multicolumn{5}{|l|}{ TNM stage } \\
\hline I-II & 22 & 18 & 4 & 0.014 \\
\hline III-IV & 77 & 40 & 37 & \\
\hline
\end{tabular}

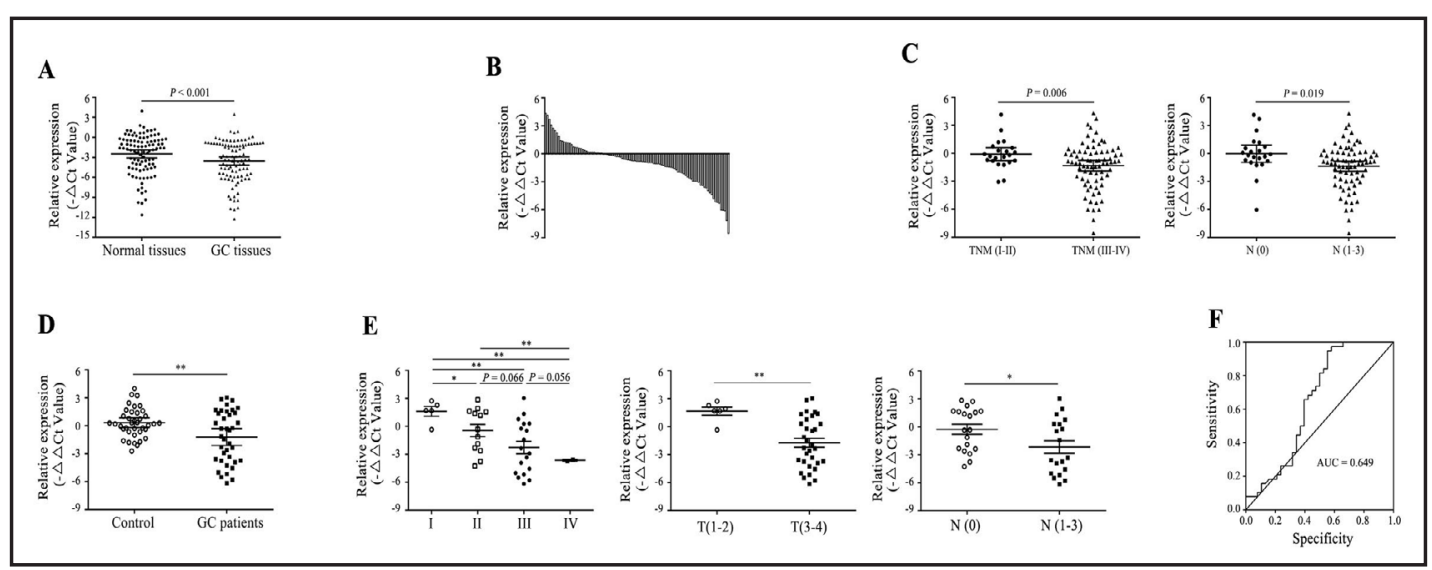

Fig. 1. The expression of MT1JP in tumor tissues and plasma of GC patients. (A) RT-qPCR for the expression of MT1JP in GC tissues and adjacent normal tissues. (B) The fold change of MT1JP expression in GC tissues compared to that in the paired non-tumor tissues for each patient $(n=99)$. (C) The correlation between tissue MT1JP expression and TNM stage or lymphatic metastasis. (D) The expression levels of plasma MT1JP in GC patients $(n=34)$ and healthy controls $(n=34)$. (E) The correlation between plasma MT1JP expression and TNM stage, invasion depth and lymphatic metastasis. (F) The ROC curve for plasma MT1JP on diagnosis of GC. ${ }^{*} \mathrm{P}<0.05,{ }^{* *} \mathrm{P}<0.01$. 
a near-significant association was observed between decreased MT1JP and increased tumor size (Table 1). However, MT1JP expression was not associated with factors such as gender, age, alcohol consumption, smoking, tumor differentiation, location, venous invasion, local invasion and distant metastasis.

Non-invasive approach using circulating lncRNAs provides new alternatives for cancer detection and prognosis evaluation $[15,17,18]$. Thus, we also measured MT1JP expression in plasma samples from $34 \mathrm{GC}$ patients and paired healthy donors. Consistent with previous findings $[17,18]$, we showed good stability of MT1JP in plasma by frozen-thawed test or room-temperature retention test (data not shown). Importantly, the circulating levels of MT1JP were also significantly reduced in GC patients compared to healthy controls (Fig. 1D). Downregulation of circulating MT1JP was significantly associated with advanced TNM stage, increased $\mathrm{T}$ depth and positive lymphatic metastasis, respectively (Fig. 1E). Finally, we plotted ROC curve to assess the diagnostic value of serum MT1JP as a biomarker for GC. As shown in Fig. 1F, the area under the ROC curve (AUC) was 0.649 (95\% CI, 0.520-0.777, $P=0.026)$. These findings suggested that MT1JP expression in tissue or plasma could be a candidate predictor for GC diagnosis and staging assessment.

\section{MT1JP is a target of miR-214-3p}

Next, we detected MT1JP expression in non-tumorigenic gastric epithelial cell line GES1 and 4 GC cell lines (MGC-803, AGS, SGC-7901 and HGC-27). Our data showed that MT1JP was generally downregulated in the GC cell lines compared with that in GES-1 (Fig. 2A). Then we generated MT1JP-overexpressing plasmid and MT1JP siRNA. The effectiveness

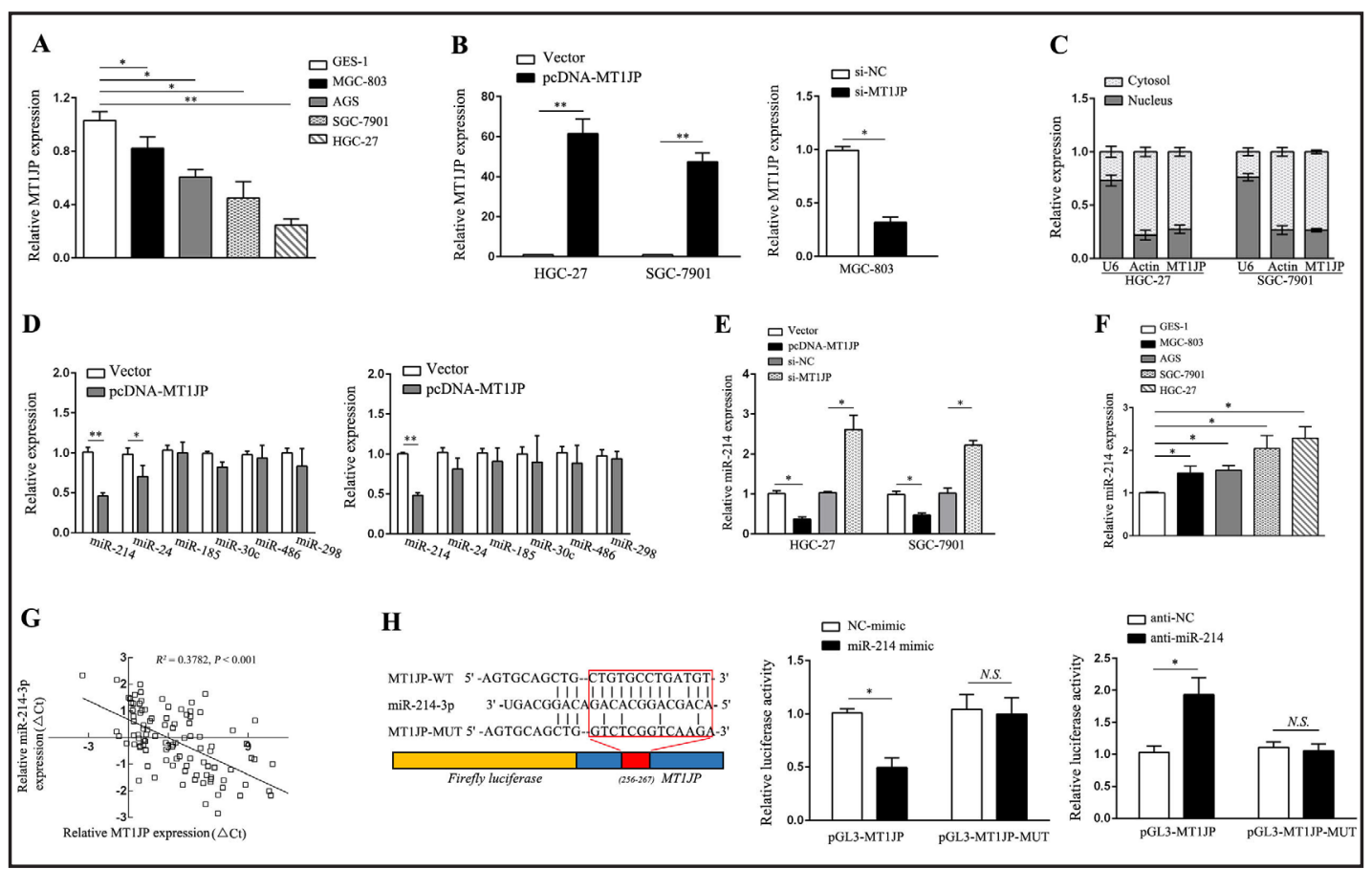

Fig. 2. MT1JP was a target of miR-214-3p. (A) The expression of MT1JP in GC cell lines. (B) Efficiency of MT1JP overexpression and knockdown. (C) The expression levels of MT1JP in nuclear and cytoplasmic fractions of GC cells. U6 (nucleus retained) and $\beta$-actin (exported to cytoplasm) were used as controls. (D) The expression of the predicted miRNAs in MT1JP-overexpressing HGC-27 and SGC-7901 cells. (E) The expression of miR-214-3p in MT1JP-overexpressing or MT1JP-knockdown HGC-27 and SGC-7901 cells. (F) The expression of miR-214-3p in GC cell lines. (G) Bivariate correlation analysis of the relationship between MT1JP and miR-214-3p levels. (H) The predicted miR-214-3p-binding sequence in MT1JP. Luciferase assays in HEK-293T cells transfected wild-type or mutant constructs of MT1JP with miR-214-3p mimics/anti-miR214-3p. ${ }^{*} \mathrm{P}<0.05$, ${ }^{* *} \mathrm{P}<0.01$. N.S. No significant difference. 
of overexpression and knockdown was shown in Fig. 2B. Generally, lncRNAs have been reported to regulate cellular processes either at the epigenetic level or at the transcriptional level. To unveil the possible mechanisms that MT1JP may involve, we learned the subcellular localization of MT1JP first. Consistent with previous findings, MT1JP was shown to be more abundant in cytoplasmic fraction than in nuclear fraction (Fig. 2C). Recently, ceRNA has been proposed as one of the major regulatory mechanisms for cytoplasm-retained lncRNAs $[19,20]$. To explore the possible ceRNA network involving MT1JP, we examined a set of miRNAs that were predicted to bind MT1JP by FINDTAR3 (https://bio.sz.tsinghua.edu.cn/) and RNAhybrid2.1 (https://bibiserv.cebitec.uni-bielefeld.de/rnahybrid/dl_pre-page.html). These candidate miRNAs included miR-214-3p, miR-24-3p, miR-185-5p, miR-30c-2-3p, miR-486-3p and miR-298. We then screened the expression profiles of these miRNAs in MT1JP-overexpressing HGC-27 and SGC-7901 cells. As shown in Fig. 2D, miR-214-3p was the most suppressed miRNA by MT1JP overexpression in both cells. miR-24-3p but not any other miRNA displayed a mild decrease by MT1JP overexpression. Thus, it was reasonable to speculate that miR-214-3p functioned as a downstream target of MT1JP. This was further supported by the findings that knockdown of MT1JP upregulated miR-214-3p levels in both cells (Fig. 2E). Moreover, MT1JP expression in GC tissues was inversely correlated with miR214-3p expression (Fig. 2G). MiR-214-3p has generally been regarded as an oncogene to promote GC tumorigenesis and progression [21-24]. Consistently, we showed that miR-2143 p expression was upregulated in 4 GC cell lines compared to GES-1 cell (Fig. 2F). To further

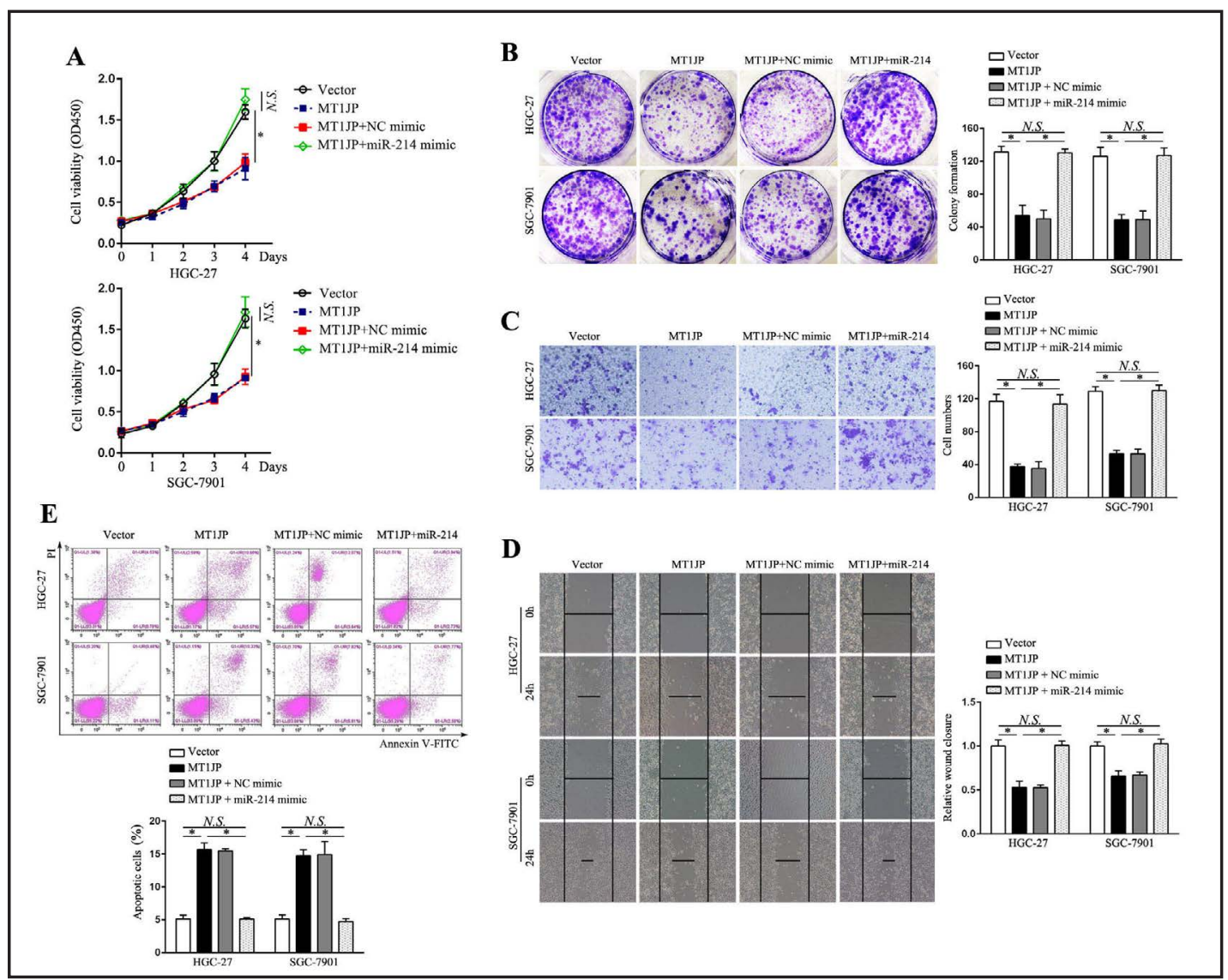

Fig. 3. MT1JP modulated GC proliferation, invasion, migration and apoptosis via negative regulation of miR214-3p. MT1JP construct and miR-214-3p mimics were co-transfected in HGC-27 and SGC-7901 cells. (A) Cell viability was detected by CCK-8 assay. (B) Colony formation. (C) Cell invasion was detected by Matrigelcoated Transwell assay. (D) Cell migration was detected by the wound healing assay. (E) Cell apoptosis was determined by flow cytometric analyses. ${ }^{*} \mathrm{P}<0.05$. N.S. No significant difference. 
clarify the underlying relationship between MT1JP and miR-214-3p, luciferase constructs containing the wild-type MT1JP transcript (pGL3-MT1JP) or MT1JP with mutated binding sequence (pGL3-MT1JP-MUT) were co-transfected into HEK-293T cell with miR-214-3p mimics or anti-miR-214-3p (Fig. 2H). The results showed that miR-214-3p reduced the luciferase activity of pGL3-MT1JP but not pGL3-MT1JP-MUT. Consistently, anti-miR-214-3p increased the luciferase activity of pGL3-MT1JP but not pGL3-MT1JP-MUT (Fig. 2H). These results indicated that miR-214-3p could bind directly to MT1JP at the miRNA recognition sites.

MT1JP modulates cell proliferation, migration, invasion and apoptosis via negative regulation of $\mathrm{miR}-214-3 p$

To investigate the effects of MT1JP on proliferation, motility and apoptosis of GC cells, CCK-8, colony formation, wound healing and Transwell assays were conducted when MT1JP was downregulated or upregulated. As shown in Fig. 3A-E, overexpression of MT1JP inhibited the proliferation, invasion, migration, but facilitated apoptosis of HGC-27 and SGC-7901 cells. MT1JP overexpression also notably suppressed Ki-67 expression in HGC-27 cells (Fig. 5C). Conversely, knockdown of MT1JP facilitated the proliferation of MGC-803 cells (data not shown). These findings suggested a tumor suppressive role of MT1JP. To further investigate whether MT1JP functioned through miR-214-3p, MT1JP-overexpressing/knockdown cells were intervened with miR-214-3p mimics or anti-miR-214-3p. As shown in Fig. 3AE, the MT1JP-induced effects on proliferation, invasion, migration and apoptosis could be abrogated by the transfection of miR-214-3p mimics in HGC-27 and SGC-7901 cells. On the contrary, anti-miR-214-3p counteracted the pro-proliferative effects by knockdown of MT1JP in MGC-803 cells (data not shown). These findings indicated that MT1JP could suppress GC cell proliferation, invasion, migration, but facilitate apoptosis via downregulation of miR214-3p.

\section{MT1JP functions as a ceRNA for miR-214-3p to facilitate RUNX3 expression}

According to bioinformatics analysis by miRWalk [25], we found that miR-214-3p could bind directly to the 3'UTR of RUNX3 with two putative complementary sequences

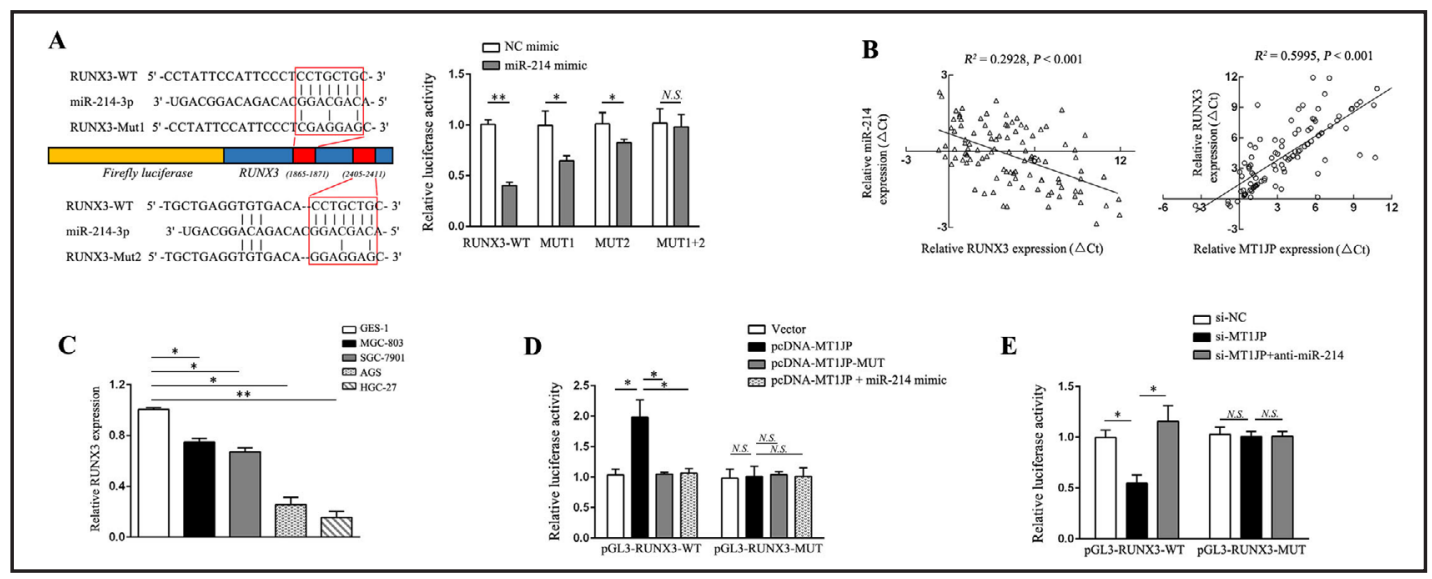

Fig. 4. MT1JP functioned as a ceRNA for miR-214-3p to facilitate RUNX3 expression. (A) Left: The putative miR-214-3p binding sites in the 3'UTR of RUNX3 (RUNX3-WT), and mutations of putative miR-2143p binding sequences in RUNX3 (MUT1, MUT2, MUT1+2). Right: Luciferase activity assay. (B) Left: The correlation between the expression levels of miR-214-3p and those of RUNX3 in GC tissues. Right: The correlation between the expression levels of MT1JP and those of RUNX3. (C) The expression levels of RUNX3 in GC cell lines. (D) Luciferase activity of pGL3 reporters which contained wild-type or mutant RUNX3 3'UTR with the indicated treatments in HEK-293T cells. (E) Luciferase activity assay. ${ }^{*} \mathrm{P}<0.05,{ }^{* *} \mathrm{P}<0.01$. N.S. No significant difference. 
(Fig. 4A). The putative binding of oncogenic miR-214-3p to RUNX3 was consistent with the tumor suppressor role of RUNX3 that has been widely reported [26-29]. Moreover, posttranscriptional repression of RUNX3 by miRNAs has recently been proposed as one of the major mechanisms resulting in RUNX3 inactivation [30-33]. To determine the functionality of these predicted sites, luciferase reporter assay with constructs containing the putative binding sequences (RUNX3-WT) or mutated types (RUNX3-MUT1: nt 1865-1871, RUNX3MUT2: nt 2405-2411, and double mutation: RUNX3-MUT1+2) was performed. As shown in Fig. 4A, miR-214-3p mimics inhibited luciferase activity of RUNX3-WT. Single mutation with either MUT1 or MUT2 partially ablated its interaction with miR-214-3p. Double mutation with MUT1+2 completely abolished this interaction. These results suggested that miR214-3p could bind to the transcript position (1865-1871 and 2405-2411) of RUNX3 3'UTR. Supporting this, RT-qPCR and Western blot results showed that transfection of miR-214-3p mimics significantly suppressed the mRNA and protein level of RUNX3 in HGC-27 and SGC7901 cells (data not shown).

Through the shared miRNA response elements (MRE), MT1JP may act as a sponge for miR-214-3p to mediate RUNX3 expression. To test this, the correlation between MT1JP, miR214-3p and RUNX3 in GC tissues were explored. As shown in Fig. 4B, the expression of RUNX3 was negatively correlated with that of miR-214-3p, but positively correlated with that of MT1JP in GC tissues. Additionally, reduced expression of RUNX3 was also detected in 4 GC cells compared with that in GES-1 cell (Fig. 4C). Next, we investigated whether the MT1JPmediated sequestration of miR-214-3p was responsible for the upregulation of RUNX3. As shown in Fig. 4D, overexpression of MT1JP but not MT1JP-MUT enhanced the luciferase activity of RUNX3-WT reporters, whereas either miR-214-3p mimics or mutation of the MRE sequences in RUNX3 abolished this effect. Consistently, knockdown of MT1JP showed the reversed effect on the luciferase activity of RUNX3, which was rescued by anti-miR-214-

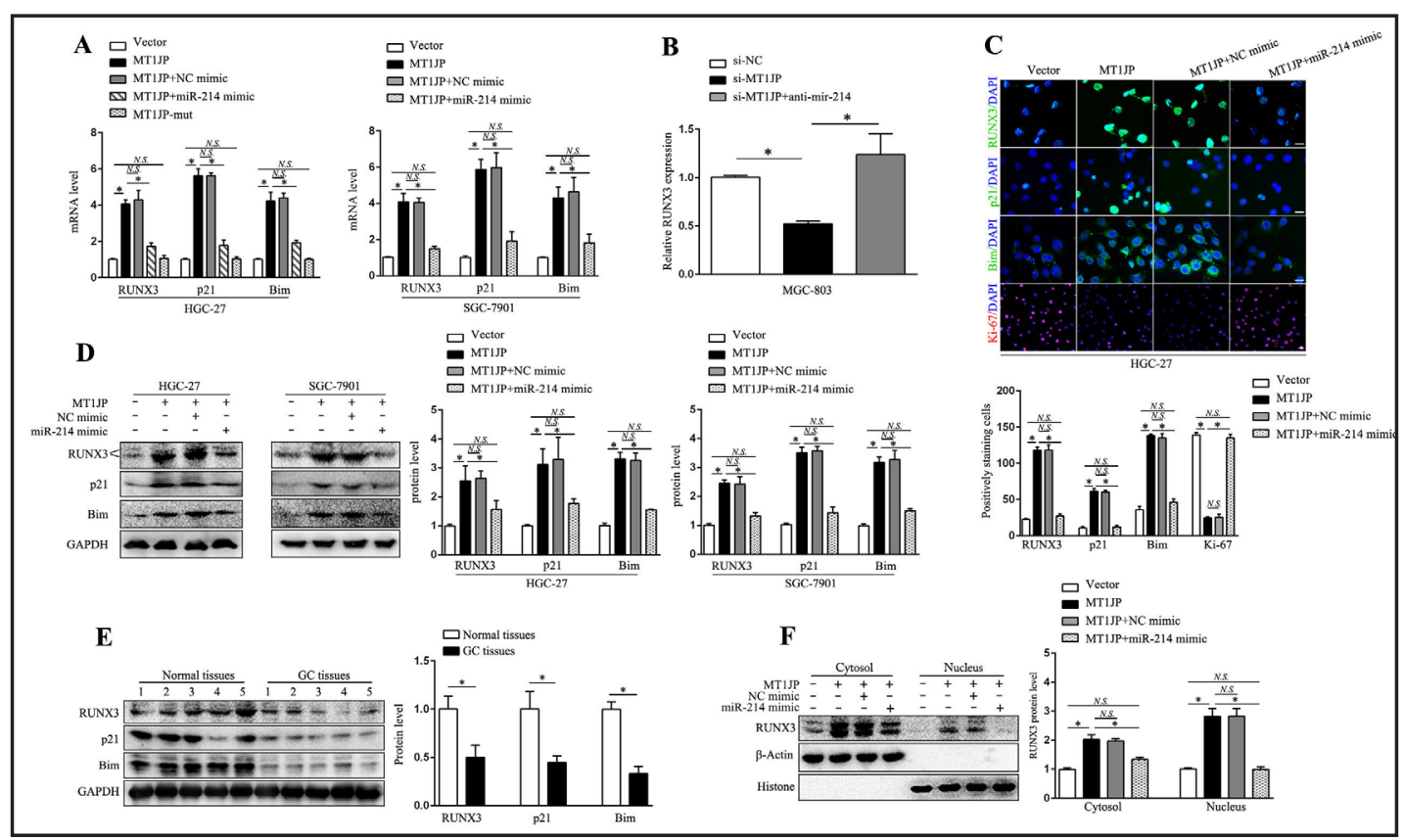

Fig. 5. MT1JP activated RUNX3 downstream signaling via negative regulation of miR-214-3p. MT1JP and miR-214-3p were co-transfected in HGC-27 and SGC-7901 cells. The RT-qPCR (A), immunostainings, scale bar: $10 \mu \mathrm{m}(\mathrm{C})$, and Western blot (D) analyses for RUNX3, p21 and Bim. (B) MT1JP siRNA and antimiR-214-3p were co-transfected in MGC-803 cells. mRNA levels of RUNX3 were detected by RT-qPCR. (E) Representative blots of RUNX3, p21 and Bim in 5 paired GC tissues and adjacent non-tumor tissues. (F) Cytoplasmic and nuclear fractions of RUNX3 in HGC-27 cells transfected MT1JP with miR-214-3p mimics. * $\mathrm{P}<0.05$. N.S. No significant difference. 
3p (Fig. 4E). These results were further confirmed at RUNX3 mRNA level and protein level (Fig. 5A-D). Previous studies have demonstrated that mislocalization of RUNX3 protein from the nucleus to the cytoplasm also contributes to inactivation of RUNX3 [28, 34]. We then analyzed the cytoplasmic and nuclear fraction of RUNX3 after MT1JP overexpression. Our results showed that MT1JP increased RUNX3 levels both at cytoplasmic and nuclear fractions, whereas miR-214-3p blocked this effect (Fig. 5F). Collectively, these results suggested that MT1JP could act as a ceRNA for miR-214-3p to facilitate RUNX3 expression in GC cells.

The MT1JP/miR-214-3p/RUNX3 axis functions as an important player in GC progression

Previous studies have indicated that $\mathrm{p} 21$ and Bim are direct downstream targets of RUNX3 that activate RUNX3-dependent apoptosis and inhibition of proliferation $[35,36]$. Consistently, our results showed that the protein levels of RUNX3, p21 and Bim concordantly decreased in GC tissues compared to paired non-cancerous tissues (Fig. 5E). In particular, overexpression of MT1JP increased both the mRNA and the protein levels of p21 and Bim in HGC-27 and SGC-7901 cells, whereas miR-214-3p mimics abolished this effect (Fig. 5A-D). We then asked whether MT1JP inhibited GC progression through RUNX3 signaling pathway. To test this, MT1JP-overexpressing GC cells were intervened with knockdown of RUNX3. The results showed that knockdown of RUNX3 is highly like miR-214-3p that completely abrogated the effects of MT1JP upregulation on the proliferation, migration, invasion and apoptosis in HGC-27 and SGC-7901 cells (Fig. 6A-E). Also, the increases in mRNA and protein levels of RUNX3, p21 and Bim by MT1JP overexpression were reversed by RUNX3 knockdown (Fig. 6F \& G). Finally, the role of MT1JP in GC tumorigenesis was analyzed in vivo and the

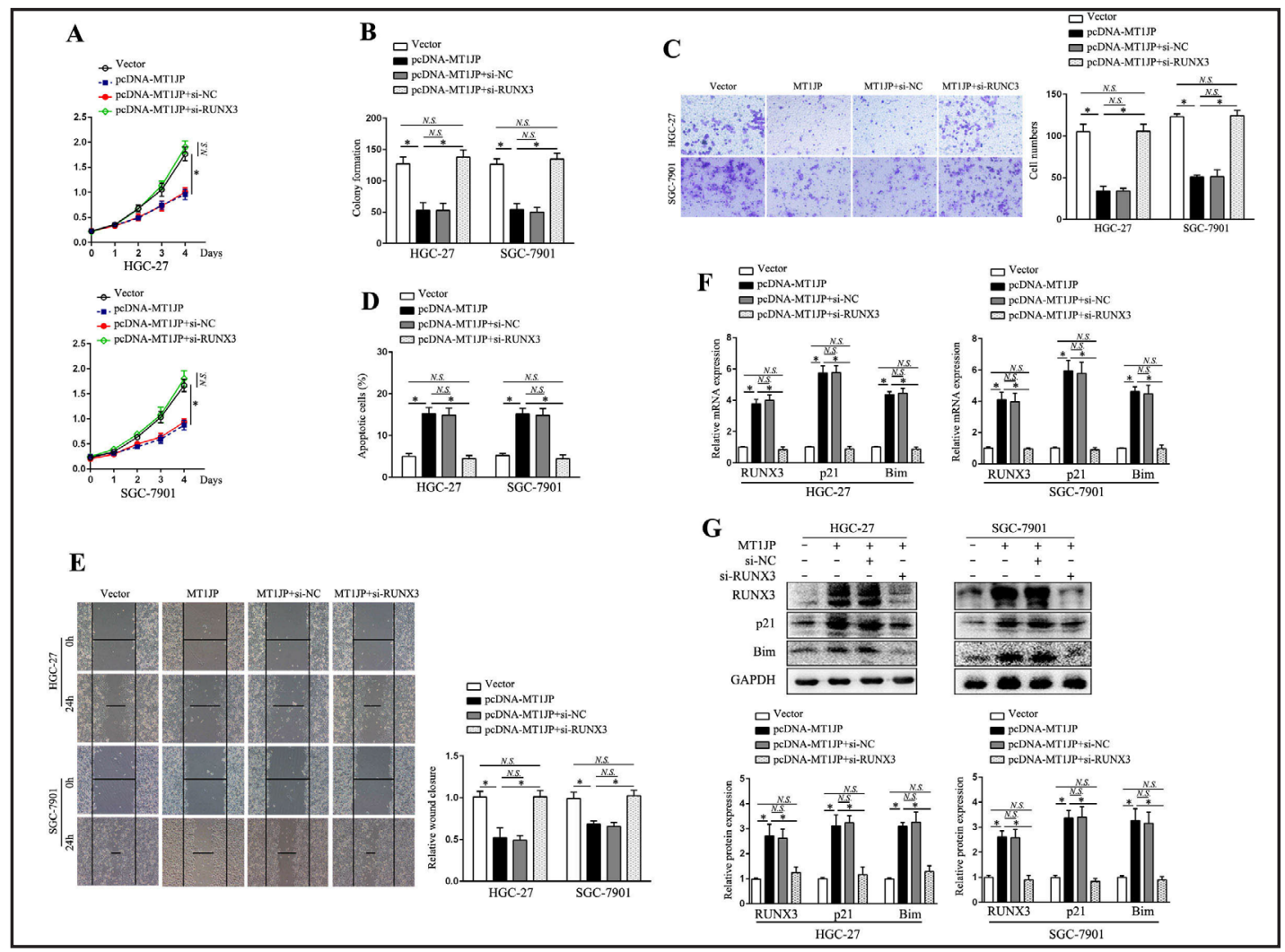

Fig. 6. The tumor suppressive effects by MT1JP overexpression could be abrogated by knockdown of RUNX3. MT1JP-overexpressing plasmid and RUNX3 siRNA were co-transfected in HGC-27 and SGC-7901 cells. Cell viability (A), colony formation (B), cell invasion (C), cell apoptosis (D), and cell migration (E) were examined, respectively. mRNA levels (F) and protein levels (G) of RUNX3, p21 and Bim were detected. * $\mathrm{P}<0.05$. N.S. No significant difference.

\section{KARGER}




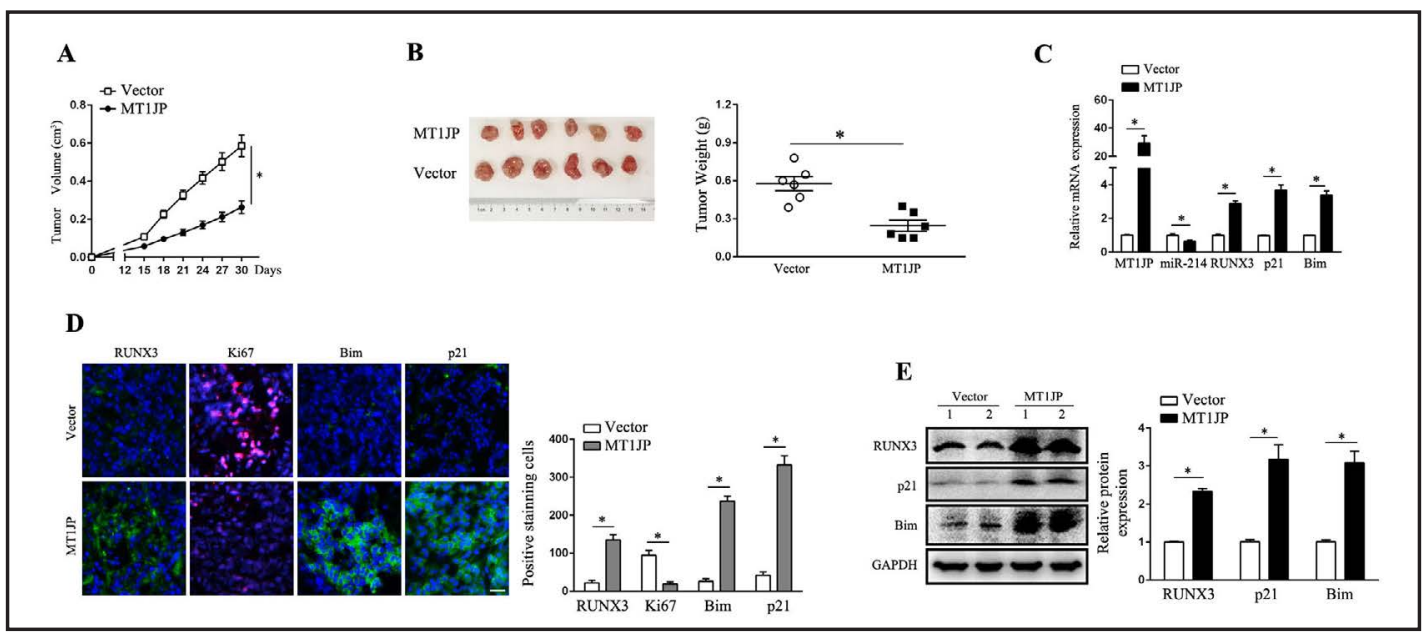

Fig. 7. The MT1JP/miR-214-3p/RUNX3 axis functioned as an important player in GC progression in vivo. (A) Tumor volume was evaluated for 15-30 days after implantation. (B) Tumor formation and tumor weight in Vector and MT1JP groups, respectively ( $\mathrm{n}=6$ for each group). (C) The transcript levels of MT1JP, miR-2143p, RUNX3, p21 and Bim. (D) Immunostainings of RUNX3, Ki-67, Bim and p21 in frozen tissue sections. Scale bar: $30 \mu \mathrm{m}$. (E) Representative blots of RUNX3, p21 and Bim in Vector and MT1JP groups. * $\mathrm{P}<0.05$.

results showed that overexpression of MT1JP significantly inhibited tumor growth (Fig. 7A\&B). MT1JP overexpression increased the expression of RUNX3, p21, Bim, but decreased the expression of miR-214-3p (Fig. 7C-E). Consistently, overexpression of MT1JP reduced the staining intensity of proliferation antigen Ki-67 (Fig. 7D). Taken together, these data suggested that MT1JP could inhibit GC progression via activating RUNX3 signaling pathway.

\section{Discussion}

Recent studies of the human transcriptome have attested that an astounding number of lncRNAs might have key roles for the transcriptional regulation linked to cancer progression $[7-9,13]$. Dysregulated lncRNA expression is documented in various cancers, and their tissuespecificity makes them attractive candidates as diagnostic/prognostic biomarkers and/or therapeutic agents. In this study, we showed that MT1JP was significantly downregulated in GC tissues/cells compared to the adjacent non-tumor tissues/normal gastric epithelial cell line, and the reduced expression of MT1JP was correlated with advanced TNM stage and lymphatic metastasis. We also analyzed the expression of MT1JP in the plasma of GC patients. Again, MT1JP was significantly decreased in the plasma of GC patients compared to that in healthy controls. Low expression of plasma MT1JP was also correlated with advanced TNM stage, increased T depth, and positive lymphatic metastasis. Given the simplicity and accuracy of plasma MT1JP detection, it may be used as an easier and promising approach for GC diagnosis and staging assessment.

MT1JP has previously been shown to act as a tumor suppressor [16]. Supporting this, we found that overexpression of MT1JP resulted in proliferation suppression, migration reduction, invasion inhibition and apoptosis induction, as opposed to the effects of MT1JP knockdown. Mechanistically, lncRNAs often function in highly complex regulatory networks because they are able to interact with proteins, RNAs, or genomic DNA [4, 5, 14, 20]. Accordingly, MT1JP has previously been reported to interact with an RNA-binding protein TIAR, thereby affecting the translational activity of tumor suppressor p53 as well as the p53related pathways [16]. CeRNA hypothesis is an emerging theory, which describes potential communication networks among all transcript RNA species mediated by miRNAs and MREs within RNA transcripts [19]. CeRNAs sequester miRNAs to regulate mRNA transcripts 
containing MRE and protect the target RNAs from repression. We showed that MT1JP was more abundant in cytoplasm rather than nucleus. This predisposed MT1JP to function as a potential ceRNA. Then we confirmed the direct binding between MT1JP and miR-214-3p using bioinformatics analysis and reported assay. Supporting this, we further found that the expression of miR-214-3p was negatively correlated with that of MT1JP expression in GC tissues. In particular, we demonstrated that the tumor suppressive effect of MT1JP on proliferation, migration and apoptosis could be reversed by the transfection of miR-214-3p mimics, whereas the pro-oncogenic effect by MT1JP knockdown could be rescued by antimiR-214-3p. Therefore, our findings provide evidence of a novel ceRNA regulatory network, where MT1JP and miR-214-3p negatively regulate each other.

MiR-214-3p is generally documented as a key oncogene in a variety of prevalent cancers including GC $[23,24,37]$. Previous studies have shown that miR-214-3p is commonly upregulated in GC tissue-derived mesenchymal stem cells and GC cancer tissues [38]. Upregulation of miR-214-3p is tightly associated with clinical progression and poor prognosis independent of clinical covariates [22]. Additionally, hemolysis-free miR-214-3p levels in plasma can also distinguish GC patients from healthy controls, monitor tumor dynamics and predict distant metastasis [21]. Knockdown of miR-214-3p inhibits proliferation and metastasis of GC cells [23, 24]. Mechanistically, miR-214-3p's pleiotropic contribution to various cancer formation is achieved via its several target genes. In fact, miR-214-3p behaves as a key hub by coordinating fundamental signaling networks such as PTEN/Akt, $\beta$-catenin, and tyrosine kinase receptor pathways $[37,39,40]$. MiR-214-3p also regulates the levels of crucial gene expression modulators, such as the epigenetic repressor EZH2, genome guardian p53, transcription factors TFAP2, and another miRNA miR-148b [41-43]. Therefore, miR-214-3p has essential roles in coordinating tumor proliferation, stemness, angiogenesis, invasiveness, extravasation, metastasis, resistance to chemotherapy, and microenvironment.

The RUNX family transcription factors are critical regulators of development and frequently dysregulated in cancer. RUNX3 has widely been described as a tumor suppressor and is involved in tumor development and metastasis at different levels, such as Epithelialmesenchymal transition (EMT), adhesion, invasion, apoptosis and angiogenesis. The gastric epithelium of RUNX3 knockout mice exhibits hyperplasia, a reduced rate of apoptosis, and reduced sensitivity to TGF- $\beta$ [26]. RUNX3 is absent or lowly expressed in $50 \%-70 \%$ primary GC and various GC cells because of hypermethylation of RUNX3 gene promoter, loss of allele heterozygosity and cytoplasmic sequestration [26-29]. In addition, miRNA has recently been highlighted as a novel regulator of RUNX3 at the post-transcriptional level [30-33]. For instance, miR-130b is proposed as a candidate for silencing RUNX3 in GC cells [33]. MiR106a targeting of RUNX3 is involved in multidrug resistance [31]. Upregulation of miR-130a and miR-495 under hypoxic conditions suppresses RUNX3 expression at the protein level through direct bindings, thereby promoting cell proliferation and tumor angiogenesis of GC cells [30]. We presented evidence that RUNX3 was a direct target of miR-214-3p. The identification of RUNX3 as a miR-214-3p target expanded the repertoire of miR-214-3p targets. In particular, we showed that the luciferase activity of RUNX3 reporters and RUNX3 mRNA expression were increased after MT1JP overexpression, but reversed by miR-214-3p mimics. However, they were reduced by knockdown of MT1JP, which could be rescued by anti-miR-214-3p. These results suggests that MT1JP functions as a ceRNA for miR-214-3p to facilitate the expression of RUNX3.

Downstream of TGF- $\beta$ signaling, RUNX3 transactivates target genes synergistically with Smads and p300 [35, 44]. Previous studies have reported p21 and Bim are direct downstream targets of RUNX3 that activated RUNX3 dependent apoptosis and inhibition of proliferation $[35,36]$. Thus, we also explored the effect of MT1JP on p21 and Bim expression. As expected, overexpressed MT1JP increased the mRNA and protein levels of p21 and Bim. This is consistent with the findings that MT1JP facilitated upregulation of nuclear RUNX3 level, a sign of RUNX3 transactivation. On the contrary, knockdown of RUNX3 reversed the MT1JPmediated upregulation of $\mathrm{p} 21$ and Bim, thereby abolishing the tumor suppressive effect of 


\begin{tabular}{|c|c|c|}
\hline Cellular Physiology & Cell Physiol Biochem 2018; & 46:2445-2459 \\
\hline and Biochemistry & $\begin{array}{l}\text { DOI: } 10.1159 / 000489651 \\
\text { Published onIIne: Thay II, } 2018\end{array}$ & $\begin{array}{l}\text { O } 2018 \text { The Author(s). Published by S. Karger AG, Basel } \\
\text { www.karger.com/cpb }\end{array}$ \\
\hline
\end{tabular}

MT1JP. Therefore, these findings implies that RUNX3 signaling acts directly downstream of MT1JP and contributes to its tumor suppressive effects.

Taken together, our study indicates that MT1JP/miR-214-3p/RUNX3 axis functions as an important player in tumorigenesis and progression of GC, and it may further promote the development of IncRNA-directed diagnosis and therapy for GC.

\section{Acknowledgements}

This study was supported by National Science Foundation of China (81500193), Natural Science Foundation of Jiangsu Province (BK20161353), the Key Youth Talents Program in Health, Jiangsu Province (QNRC2016454), China Postdoctoral Science Foundation (2015M571703), Social Development Foundation of Zhenjiang (SH2015059) and the key R\&D Special Fund of Jiangsu Province (BE2015666). The funders had no role in study design, data collection and analysis, decision to publish, or preparation of the manuscript.

\section{Disclosure Statement}

The authors declare to have no conflict of interests.

\section{References}

1 Jemal A, Bray F, Center MM, Ferlay J, Ward E, Forman D: Global cancer statistics. CA Cancer J Clin 2011;61:69-90.

-2 Nagini S: Carcinoma of the stomach: A review of epidemiology, pathogenesis, molecular genetics and chemoprevention. World J Gastrointest Oncol 2012;4:156-169.

- 3 Leung WK, Wu MS, Kakugawa Y, Kim JJ, Yeoh KG, Goh KL, Wu KC, Wu DC, Sollano J, Kachintorn U, Gotoda T, Lin JT, You WC, Ng EK, Sung JJ, Asia Pacific Working Group on Gastric C: Screening for gastric cancer in Asia: current evidence and practice. Lancet Oncol 2008;9:279-287.

4 Mercer TR, Dinger ME, Mattick JS: Long non-coding RNAs: insights into functions. Nat Rev Genet 2009;10:155-159.

-5 Wang KC, Chang HY: Molecular mechanisms of long noncoding RNAs. Mol Cell 2011;43:904-914.

6 Geisler S, Coller J: RNA in unexpected places: long non-coding RNA functions in diverse cellular contexts. Nat Rev Mol Cell Biol 2013;14:699-712.

7 Huarte M: The emerging role of lncRNAs in cancer. Nat Med 2015;21:1253-1261.

8 Iyer MK, Niknafs YS, Malik R, Singhal U, Sahu A, Hosono Y, Barrette TR, Prensner JR, Evans JR, Zhao S, Poliakov A, Cao X, Dhanasekaran SM, Wu YM, Robinson DR, Beer DG, Feng FY, Iyer HK, Chinnaiyan AM: The landscape of long noncoding RNAs in the human transcriptome. Nat Genet 2015;47:199-208.

-9 Sun W, Yang Y, Xu C, Xie Y, Guo J: Roles of long noncoding RNAs in gastric cancer and their clinical applications. J Cancer Res Clin Oncol 2016;142:2231-2237.

$>10$ Li L, Geng Y, Feng R, Zhu Q, Miao B, Cao J, Fei S: The Human RNA Surveillance Factor UPF1 Modulates Gastric Cancer Progression by Targeting Long Non-Coding RNA MALAT1. Cell Physiol Biochem 2017;42:2194-2206.

11 Yan J, Zhang Y, She Q, Li X, Peng L, Wang X, Liu S, Shen X, Zhang W, Dong Y, Lu J, Zhang G: Long Noncoding RNA H19/miR-675 Axis Promotes Gastric Cancer via FADD/Caspase 8/Caspase 3 Signaling Pathway. Cell Physiol Biochem 2017;42:2364-2376.

12 Yan K, Tian J, Shi W, Xia H, Zhu Y: LncRNA SNHG6 is Associated with Poor Prognosis of Gastric Cancer and Promotes Cell Proliferation and EMT through Epigenetically Silencing p27 and Sponging miR-101-3p. Cell Physiol Biochem 2017;42:999-1012.

13 Chandra Gupta S, Nandan Tripathi Y: Potential of long non-coding RNAs in cancer patients: From biomarkers to therapeutic targets. Int J Cancer 2017;140:1955-1967. 


\section{Cellular Physiology Cell Physiol Biochem 2018;46:2445-2459

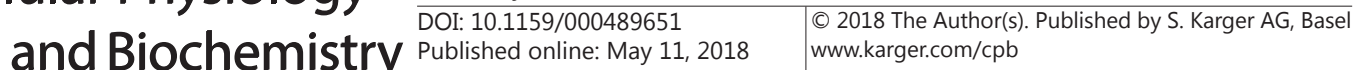

Xu et al.: MT1JP Regulates GC through miR-214-3p/RUNX3

14 Yoon JH, Abdelmohsen K, Srikantan S, Yang X, Martindale JL, De S, Huarte M, Zhan M, Becker KG, Gorospe M: LincRNA-p21 suppresses target mRNA translation. Mol Cell 2012;47:648-655.

15 Huang YK, Yu JC: Circulating microRNAs and long non-coding RNAs in gastric cancer diagnosis: An update and review. World J Gastroenterol 2015;21:9863-9886.

16 Liu L, Yue H, Liu Q, Yuan J, Li J, Wei G, Chen X, Lu Y, Guo M, Luo J, Chen R: LncRNA MT1JP functions as a tumor suppressor by interacting with TIAR to modulate the p53 pathway. Oncotarget 2016;7:1578715800.

17 Huang R, Wang X, Zhang W, Zhangyuan G, Jin K, Yu W, Xie Y, Xu X, Wang H, Sun B: Down-Regulation of LncRNA DGCR5 Correlates with Poor Prognosis in Hepatocellular Carcinoma. Cell Physiol Biochem 2016;40:707-715.

18 Tong YS, Wang XW, Zhou XL, Liu ZH, Yang TX, Shi WH, Xie HW, Lv J, Wu QQ, Cao XF: Identification of the long non-coding RNA POU3F3 in plasma as a novel biomarker for diagnosis of esophageal squamous cell carcinoma. Mol Cancer 2015;14:3.

19 Salmena L, Poliseno L, Tay Y, Kats L, Pandolfi PP: A ceRNA hypothesis: the Rosetta Stone of a hidden RNA language? Cell 2011;146:353-358.

20 Tay Y, Rinn J, Pandolfi PP: The multilayered complexity of ceRNA crosstalk and competition. Nature 2014;505:344-352.

21 Zhang KC, Xi HQ Cui JX, Shen WS, Li JY, Wei B, Chen L: Hemolysis-free plasma miR-214 as novel biomarker of gastric cancer and is correlated with distant metastasis. Am J Cancer Res 2015;5:821-829.

22 Ueda T, Volinia S, Okumura H, Shimizu M, Taccioli C, Rossi S, Alder H, Liu CG, Oue N, Yasui W, Yoshida K, Sasaki H, Nomura S, Seto Y, Kaminishi M, Calin GA, Croce CM: Relation between microRNA expression and progression and prognosis of gastric cancer: a microRNA expression analysis. Lancet Oncol 2010;11:136146.

23 Xin R, Bai F, Feng Y, Jiu M, Liu X, Bai F, Nie Y, Fan D: MicroRNA-214 promotes peritoneal metastasis through regulating PTEN negatively in gastric cancer. Clin Res Hepatol Gastroenterol 2016;40:748-754.

24 Yang TS, Yang XH, Wang XD, Wang YL, Zhou B, Song ZS: MiR-214 regulate gastric cancer cell proliferation, migration and invasion by targeting PTEN. Cancer Cell Int 2013;13:68.

25 Dweep H, Gretz N: miRWalk2.0: a comprehensive atlas of microRNA-target interactions. Nat Methods 2015;12:697.

26 Li QL, Ito K, Sakakura C, Fukamachi H, Inoue K, Chi XZ, Lee KY, Nomura S, Lee CW, Han SB, Kim HM, Kim WJ, Yamamoto H, Yamashita N, Yano T, Ikeda T, Itohara S, Inazawa J, Abe T, Hagiwara A, Yamagishi H, Ooe A, Kaneda A, Sugimura T, Ushijima T, Bae SC, Ito Y: Causal relationship between the loss of RUNX3 expression and gastric cancer. Cell 2002;109:113-124.

27 Sakakura C, Hasegawa K, Miyagawa K, Nakashima S, Yoshikawa T, Kin S, Nakase Y, Yazumi S, Yamagishi H, Okanoue T, Chiba T, Hagiwara A: Possible involvement of RUNX3 silencing in the peritoneal metastases of gastric cancers. Clin Cancer Res 2005;11:6479-6488.

28 Ito K, Liu Q, Salto-Tellez M, Yano T, Tada K, Ida H, Huang C, Shah N, Inoue M, Rajnakova A, Hiong KC, Peh BK, Han HC, Ito T, Teh M, Yeoh KG, Ito Y: RUNX3, a novel tumor suppressor, is frequently inactivated in gastric cancer by protein mislocalization. Cancer Res 2005;65:7743-7750.

-29 Peng Z, Wei D, Wang L, Tang H, Zhang J, Le X, Jia Z, Li Q, Xie K: RUNX3 inhibits the expression of vascular endothelial growth factor and reduces the angiogenesis, growth, and metastasis of human gastric cancer. Clin Cancer Res 2006;12:6386-6394.

-30 Lee SH, Jung YD, Choi YS, Lee YM: Targeting of RUNX3 by miR-130a and miR-495 cooperatively increases cell proliferation and tumor angiogenesis in gastric cancer cells. Oncotarget 2015;6:33269-33278.

-31 Zhang Y, Lu Q, Cai X: MicroRNA-106a induces multidrug resistance in gastric cancer by targeting RUNX3 FEBS Lett 2013;587:3069-3075.

32 Kitago M, Martinez SR, Nakamura T, Sim MS, Hoon DS: Regulation of RUNX3 tumor suppressor gene expression in cutaneous melanoma. Clin Cancer Res 2009;15:2988-2994.

33 Lai KW, Koh KX, Loh M, Tada K, Subramaniam MM, Lim XY, Vaithilingam A, Salto-Tellez M, Iacopetta B, Ito Y, Soong R, Singapore Gastric Cancer C: MicroRNA-130b regulates the tumour suppressor RUNX3 in gastric cancer. Eur J Cancer 2010;46:1456-1463.

-34 Subramaniam MM, Chan JY, Soong R, Ito K, Yeoh KG, Wong R, Guenther T, Will O, Chen CL, Kumarasinghe MP, Ito Y, Salto-Tellez M: RUNX3 inactivation in colorectal polyps arising through different pathways of colonic carcinogenesis. Am J Gastroenterol 2009;104:426-436. 


\section{Cellular Physiology Cell Physiol Biochem 2018;46:2445-2459

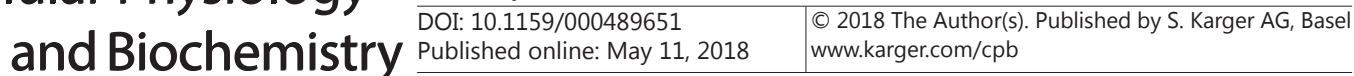

Xu et al.: MT1JP Regulates GC through miR-214-3p/RUNX3

35 Chi XZ, Yang JO, Lee KY, Ito K, Sakakura C, Li QL, Kim HR, Cha EJ, Lee YH, Kaneda A, Ushijima T, Kim WJ, Ito Y, Bae SC: RUNX3 suppresses gastric epithelial cell growth by inducing p21(WAF1/Cip1) expression in cooperation with transforming growth factor \{beta\}-activated SMAD. Mol Cell Biol 2005;25:8097-8107.

-36 Yano T, Ito K, Fukamachi H, Chi XZ, Wee HJ, Inoue K, Ida H, Bouillet P, Strasser A, Bae SC, Ito Y: The RUNX3 tumor suppressor upregulates Bim in gastric epithelial cells undergoing transforming growth factor betainduced apoptosis. Mol Cell Biol 2006;26:4474-4488.

-37 Penna E, Orso F, Taverna D: miR-214 as a key hub that controls cancer networks: small player, multiple functions. J Invest Dermatol 2015;135:960-969.

-38 Wang M, Zhao C, Shi H, Zhang B, Zhang L, Zhang X, Wang S, Wu X, Yang T, Huang F, Cai J, Zhu Q Zhu W, Qian H, Xu W: Deregulated microRNAs in gastric cancer tissue-derived mesenchymal stem cells: novel biomarkers and a mechanism for gastric cancer. Br J Cancer 2014;110:1199-1210.

39 Wang YS, Wang YH, Xia HP, Zhou SW, Schmid-Bindert G, Zhou CC: MicroRNA-214 regulates the acquired resistance to gefitinib via the PTEN/AKT pathway in EGFR-mutant cell lines. Asian Pac J Cancer Prev 2012;13:255-260.

40 Xia H, Ooi LL, Hui KM: MiR-214 targets beta-catenin pathway to suppress invasion, stem-like traits and recurrence of human hepatocellular carcinoma. PLoS One 2012;7:e44206.

41 Derfoul A, Juan AH, Difilippantonio MJ, Palanisamy N, Ried T, Sartorelli V: Decreased microRNA-214 levels in breast cancer cells coincides with increased cell proliferation, invasion and accumulation of the Polycomb Ezh2 methyltransferase. Carcinogenesis 2011;32:1607-1614.

42 Xu CX, Xu M, Tan L, Yang H, Permuth-Wey J, Kruk PA, Wenham RM, Nicosia SV, Lancaster JM, Sellers TA, Cheng JQ: MicroRNA miR-214 regulates ovarian cancer cell stemness by targeting p53/Nanog. J Biol Chem 2012;287:34970-34978.

43 Penna E, Orso F, Cimino D, Vercellino I, Grassi E, Quaglino E, Turco E, Taverna D: miR-214 coordinates melanoma progression by upregulating ALCAM through TFAP2 and miR-148b downmodulation. Cancer Res 2013;73:4098-4111.

44 Iwatani K, Fujimoto T, Ito T: Cyclin D1 blocks the anti-proliferative function of RUNX3 by interfering with RUNX3-p300 interaction. Biochem Biophys Res Commun 2010;400:426-431. 